LA-UR-95-2091

\title{
Classical Dynamics for Linear Systems: The Case of Quantum Brownian Motion
}

\author{
James Anglin ${ }^{\star}$ and Salman Habib H $^{\dagger}$ \\ Theoretical Division \\ Mail Stop B288 \\ Los Alamos National Laboratory \\ Los Alamos, NM 87545
}

\begin{abstract}
It has long been recognized that the dynamics of linear quantum systems is classical in the Wigner representation. Yet many conceptually important linear problems are typically analyzed using such generally applicable techniques as influence functionals and Bogoliubov transformations. In this Letter we point out that the classical equations of motion provide a simpler and more intuitive formalism for linear quantum systems. We examine the important problem of Brownian motion in the independent oscillator model, and show that the quantum dynamics is described directly and completely by a c-number Langevin equation. We are also able to apply recent insights into quantum Brownian motion to show that the classical Fokker-Planck equation is always local in time, regardless of the spectral density of the environment.
\end{abstract}

e-mail:

*anglin@t6-serv.lanl.gov

†habib@predator.lanl.gov 
It has long been recognized that, although the Wigner function of a linear quantum mechanical system cannot in general be considered as a classical ensemble density, yet it evolves according to exactly the same Liouville equation as the corresponding classical system: $\dot{f}=\hat{L}_{c l} f$. [1] This is true even for linear systems with time-dependent parameters, and for non-linear systems treated in Gaussian approximation [2]. Nevertheless, conceptually important problems, such as quantum Brownian motion and particle production in classical background fields, are generally treated using formalisms which do not explicitly take advantage of classicality, such as path integrals and Bogoliubov transformations. An important example of the disadvantages of this unnecessary sophistication is the way in which the machinery of influence functionals has successfully hidden the fact that, in the independent oscillator model [3], quantum and classical Brownian motion are dynamically identical.

The more sophisticated approaches are often technically cumbersome, and their interpretation frequently seems rather subtle. It is usually a challenge, for example, to associate a simple physical intuition with the closed time paths of an influence functional. In contrast, a much more direct approach to any linear problem is to use the classical equations of motion to obtain the time evolution of the Wigner function. After solving these equations, with their (usually easiest) Cauchy boundary conditions, one has nothing further to do (unless one is studying an open system, in which case there remains the integration over the initial data of the environment). And since the classical trajectories of a system are easy to associate with a consistent physical intuition, one can not only compute the correct final answer, but also appreciate the intermediate processes that generate the ultimate result. Since most linear problems are essentially of conceptual interest, this is certainly a consummation to be wished: Classical mechanics can greatly simplify quantum mechanics.

In the linear regime, the converse can also be true. It is widely assumed that, even with the simple environmental model of independent oscillators, the classical Fokker-Planck equation for a Brownian oscillator is only local in time if one assumes a particular spectral density for the environmental bath. This is not true. By applying an insight from a recent solution of the quantum problem, we can abandon the Markovian assumption in classical Brownian motion, and still obtain a local Fokker-Planck equation. In the non-Markovian regime, the correct identification of the physical noise and the effective application of the fluctuation-dissipation relation become much more complicated [4. Without the use of methods inspired by quantum mechanics, it would be very difficult indeed to discern the Fokker-Planck equation: In this problem, quantum mechanics can greatly simplify classical mechanics. Moreover, the demonstration that the classical problem is indeed dynamically identical to 
the quantum problem must remove any lingering suspicion that influence functionals (or any other essentially quantum techniques) are necessary to capture the quantum dynamics.

To proceed to explicit details, we recall the Hamiltonian for the Brownian harmonic oscillator linearly coupled to a bath of independent oscillators:

$$
H=\frac{1}{2 m} P^{2}+\frac{m}{2} \Omega^{2} Q^{2}+\frac{1}{2} \int_{0}^{\infty} d \omega\left[p_{\omega}^{2}+\left(\omega q_{\omega}-g_{\omega} x\right)^{2}\right],
$$

where $g_{\omega}$ is the coupling constant, whose $\omega$-dependence effectively incorporates the spectral density of the environment. We also recall the Liouville propagator for the probability density in phase space:

$$
\begin{aligned}
& f\left(Q_{F}, P_{F} ;\left\{q_{\omega F}, p_{\omega F}\right\} ; t\right)=\int d Q_{I} d P_{I} D q_{I} D p_{I} \delta\left(P_{F}-P(t)\right) \delta\left(Q_{F}-Q(t)\right) \\
& \times \int \prod_{\omega} \delta\left(q_{\omega F}-q_{\omega}(t)\right) \delta\left(p_{\omega F}-p_{\omega}(t)\right) \\
& \times f\left(Q_{I}, P_{I} ;\left\{q_{\omega I}, p_{\omega I}\right\}, t_{I}\right)
\end{aligned}
$$

where $Q(t), q_{\omega}(t)$ and $P(t), p_{\omega}(t)$ denote the values of the canonical variables at time $t$ after evolution, under Hamilton's equations, from the initial values $Q_{I}, P_{I}$ and $\left\{q_{\omega I}, p_{\omega I}\right\}$ at time $t_{I}$. We write $Q(t)$ instead of $Q\left(t ; Q_{I}, P_{I},\left\{q_{\omega I}, p_{\omega I}\right\}\right)$, etc., only in order to avoid an unwieldy notation. It will be important to remember, despite this shorthand convention, that $Q(t)$ and $P(t)$ are time-dependent linear combinations of all the initial variables.

As in the standard theoretical version of Brownian motion, we assume that the intitial ensemble factorizes and that its environmental part is Gaussian円:

$$
f\left(Q_{I}, P_{I} ;\left\{q_{I}(\omega), p_{I}(\omega)\right\}, t_{I}\right)=\bar{f}\left(Q_{I}, P_{I} ; t_{I}\right) \prod_{\omega}\left(\frac{\omega \beta_{\omega}}{2 \pi}\right) e^{-\frac{1}{2} \beta_{\omega}\left[\omega^{2} q_{\omega I}^{2}+p_{\omega I}^{2}\right]} .
$$

Since we wish to know only the reduced distribution $\bar{f}(Q, P ; t)$ over the observed sector of phase space, we integrate over the environmental sector in (2). With the factorized initial condition (3), this is equivalent to propagating $\bar{f}$ using the equations of motion $P(t)=m \dot{Q}(t)$ and

$$
\ddot{Q}(t)+\Omega^{2} Q(t)+K(t) Q_{I}+\int_{0}^{t} d t^{\prime} K\left(t-t^{\prime}\right) \dot{Q}\left(t^{\prime}\right)=\frac{F(t)}{m},
$$

where $K(t) \equiv(1 / m) \int_{0}^{\infty} d \omega g_{\omega}^{2} \cos \omega t$, and $F(t)$ is a stochastic force. Since the environmental part of (3) is Gaussian, $F$ is completely described by its two-point correlation

\footnotetext{
${ }^{1}$ Slight variations in this initial distribution may be contemplated, as may variations in the Hamiltonian that are obtained by canonical transformations mixing the system and environmental sectors. These variations can be encompassed by straightforward generalizations of the present discussion.
} 
function:

$$
\left\langle F(t) F\left(t^{\prime}\right)\right\rangle=\int_{0}^{\infty} d \omega \frac{g_{\omega}^{2}}{\beta_{\omega}} \cos \omega\left(t-t^{\prime}\right) .
$$

Eqn. (4) is the (generalized) Langevin equation. The standard classical approach to this problem relies on separating the systematic and stochastic terms in this equation [4]. In the special case where $\pi g_{\omega}^{2} \equiv 4 m \gamma$ is constant, the integral kernel $K$ becomes a delta function, and one obtains a stochastic differential equation with Ohmic damping. The entire LHS of (田) is then systematic, and the RHS can be unambiguously identified as the noise. The usual classical prescription $\beta_{\omega}=\left(k_{B} T\right)^{-1}$, independent of $\omega$ as well, also ensures that the environmental noise is white. In this case, it is easy to use the fluctuation-dissipation relation $\left\langle F(t) F\left(t^{\prime}\right)\right\rangle=4 m \gamma k_{B} T \delta\left(t-t^{\prime}\right)$ to derive an evolution equation for the reduced phase space distribution $\bar{f}$, which is local in time: the Fokker-Planck equation.

For general $g_{\omega}$, however, Langevin equations are integro-differential, incorporating a back-reaction term with memory. The noise is also colored in general (i.e., the correlation function is not a delta function), even with constant $\beta_{\omega}$. One might think that these circumstances would prevent the generalized Fokker-Planck equation from being local. In fact, because the memory term depends on the past history of $\dot{Q}$, over all of which it has been affected by the environmental noise, it actually contains an implicit stochastic part. So the physical noise receives a contribution from back-reaction, as well as the explicit "bare noise." The crucial identification of the systematic and stochastic parts of the Langevin equation is therefore more subtle.

One can still derive a generalized Fokker-Planck equation by the standard method, using the colored physical noise that includes back-reaction, and the non-local systematic evolution. Remarkably, it will turn out that a rather complicated kind of fluctuation-dissipation relation can be found, which will guarantee that the generalized Fokker-Planck equation derived from Eqn. (4) will always be local in time [5]. But this approach is quite cumbersome.

A much shorter derivation is available, inspired by the quantum mechanical treatment of Ref. [6]. Integrating over the environmental sector in Eqn. (2), and using a representation for the two surviving delta functions that is more familiar in quantum contexts, we can write

$$
\begin{aligned}
& \dot{\bar{f}}\left(Q_{F}, P_{F} ; t\right)=i \int d Q_{I} d P_{I} \bar{f}\left(Q_{I}, P_{I} ; t_{I}\right) \int D d q_{I} D p_{I} \mathrm{e}^{-\frac{1}{2} \int_{0}^{\infty} d \omega \beta_{\omega}\left[\omega^{2} q_{\omega I}^{2}+p_{\omega I}^{2}\right]} \\
& \quad \times \int \frac{d k d k^{\prime}}{(2 \pi)^{2}} e^{i k\left[Q_{F}-Q(t)\right]+k^{\prime}\left[P_{F}-P(t)\right]}\left(k \dot{Q}(t)-k^{\prime} \dot{P}(t)\right) .
\end{aligned}
$$

Because the Langevin equation is linear, the solutions $Q(t), P(t)$ depend linearly on 
the initial variables $\left\{q_{\omega I}, p_{\omega I}\right\}$ and $Q_{I}, P_{I}$.

The $k$ and $k^{\prime}$ pre-factors in (6) can be replaced by derivatives under the integral with respect to $Q_{F}$ and $P_{F}$. The thus-restored delta functions can then be used to replace the $Q_{I}$ and $P_{I}$ terms in $\dot{Q}(t)$ and $\dot{P}(t)$ with linear combinations of $Q_{F}, P_{F}$, and the $q_{\omega I}$ and $p_{\omega I}$. The Gaussian integrals over the environmental variables can then be performed. The prefactors proportional to $q_{\omega I}$ and $p_{\omega I}$ lead to terms proportional to $k$ and $k^{\prime}$, which can once again be expressed as derivatives with respect to $Q_{F}$ and $P_{F}$. At the end of this process, we drop the subscripts on $Q_{F}$ and $P_{F}$, and obtain an equation of the form

$$
\dot{\bar{f}}=-\frac{P}{m} \frac{\partial \bar{f}}{\partial Q}+m \bar{\Omega}^{2}(t) Q \frac{\partial \bar{f}}{\partial P}+2 \bar{\gamma}(t) \frac{\partial}{\partial P} P \bar{f}+d(t) \frac{\partial^{2} \bar{f}}{\partial Q \partial P}+D(t) \frac{\partial^{2} \bar{f}}{\partial P^{2}} .
$$

The time-dependent co-efficients may be expressed in terms of the parameters in the Hamiltonian (11). Their calculation is straightforward but tedious, and we will not perform it here. Instead we merely note that time-dependent co-efficients, as well as the so-called "anomalous diffusion" term proportional to $d(t)$, both previously encountered in quantum treatments, do also appear in the classical problem.

If we set $\beta_{n}=\frac{2}{\hbar \omega_{n}} \tanh \frac{\hbar \omega_{n}}{2 k_{B} T}$ in (3) and (6), then equation (7) is the Hu-PazZhang master equation [7], in the Wigner representation. Any positive choice of $\beta_{n}$, however, will still provide a local equation for $\dot{\bar{f}}$. And while $\beta_{n}=\left(k_{B} T\right)^{-1}$ is prescribed by classical statistical mechanics (and for Ohmic spectra this differs from quantum mechanics in permitting white noise for all temperatures), any positive $\beta_{n}$ still defines an allowed (i.e., positive) classical ensemble density for the environment. In the case of factorized, environmentally Gaussian initial conditions, then, classical and quantum Brownian motion can be said to differ only in the constraints prescribed for $\bar{f}\left(Q_{I}, P_{I} ; t_{I}\right)$. Dynamically, the quantum and classical cases are identical.

Moving beyond the specific linear problem of Brownian motion, it is straightforward to verify that, for the most general time-dependent quadratic Hamiltonian, the propagator for the quantum mechanical Wigner function is of exactly the same form as Eqn. (2). This means that the quantum Liouville equation for a linear system in the Wigner representation will be the same as the classical Liouville equation for the corresponding classical Hamiltonian. Moreover, this means that the classical equations of motion, with their classically causal boundary conditions, provide as exact and complete an expression of linear quantum dynamics as any operator equation or path integral. And for linear open quantum systems with an initially Gaussian environment, the reduced dynamics is directly expressed in the c-number Langevin equations.

These c-number equations are both conceptually and computationally much less 
demanding than the more general formulations of quantum dynamics. If one wishes to obtain the final Wigner function $f(Q, P ; t)$ of a linear quantum system, one need only solve the time-reversed classical equations of motion to determine the initial phasespace point corresponding to the final point $(Q, P)$. The initial Wigner function at this initial point is the quantity sought. The Green's functions needed to do this for final Cauchy conditions are typically much easier to use than those required for the mixed initial and final conditions of other approaches, and since the initial Wigner function is given, one need do no further work once the classical equations are solved. And of course this program remains effective for linear systems with any number of degrees of freedom.

Furthermore, in many cases one may be able to gain considerable information from the equations of motion alone, with only qualitative discussion of the initial Wigner function. For example, knowing that a bath of independent oscillators provides a Langevin noise, one can conclude that environmental fluctuations will tend to even out oscillations in the Wigner function of a Brownian oscillator. Rapid oscillations between positive and negative values will be suppressed quickly, and this provides one of the simplest intuitive pictures of the process of decoherence (since such oscillations are the Wigner signature of interference between classically distinct pointer states).

As a second and less trivial example, we can also use classical Langevin equations to understand why so-called "supra-Ohmic" environments tend to induce little decoherence in Brownian oscillators [8] (a more complete discussion will be given elsewhere [5]). In the present formalism, supra-Ohmic environments are those for which $g_{\omega}^{2}$ increases with $\omega$ (up to some cut-off scale, generally taken to be much larger than all other scales in the problem). If one compares Ohmic and supra-Ohmic models in which $g_{\Omega}^{2}$ are equal, one typically finds that (after a few cut-off timescales) the supra-Ohmic environment produces weaker diffusion in the Brownian oscillator. This is because stronger coupling at high frequencies means that adiabatic dragging of fast environmental oscillators gives the Brownian particle a higher effective mass. Indeed, Brownian motion in supra-Ohmic environments heavily weighted in the ultraviolet rapidly becomes indistinguishable from Ohmic motion with a renormalized mass [5].

One can easily anticipate this effect by using an adiabatic approximation to write an effective Lagrangian without the fast degrees of freedom, but it is interesting to see how it appears in the exact solution of the full problem. Computing the co-efficients in the master equation (ब) merely confirms that diffusion dies away; studying the Langevin equation (4) reveals how this occurs. After solving the equation, one can always in principle re-write it in the form

$$
\ddot{Q}(t)+\tilde{\Omega}^{2}(t) Q(t)+2 \gamma(t) \dot{Q}(t)=\frac{1}{m}\left[F(t)+F_{B R}(t)\right],
$$


where in addition to the bare force $F$ (whether stochastic or systematic) there also appears the back-reaction force $F_{B R}$. In the case of a UV-dominated supra-Ohmic environment, there is a brief inertial epoch that lasts for a few cut-off times, during which the system behaves much as an Ohmic model, and the back-reaction force is small. After this epoch, however, a remarkable regime emerges in which any impulse applied to the Brownian oscillator as a bare force is rapidly echoed in the back-reaction force, with opposite sign and nearly equal magnitude [5]. This "counter-punch" effectively suppresses all forces acting on the oscillator, unless they vary rapidly on the cut-off timescale, by a large factor equal to the ratio of bare and renormalized masses found in the adiabatic analysis. Thus we achieve a nice intuitive picture, in which the supra-Ohmic environment appears to deliberately isolate the Brownian particle, and in which the importance of back-reaction is clearly exhibited.

When using classical intuition in quantum problems, one must of course be careful. Wigner functions cannot, in general, be interpreted as probability densities, because they can be negative. Even when one happens to have a positive definite Wigner function, as initially one has in the environmental sector for most Brownian motion problems, one cannot assume that the classical interpretation will persist for all times. For example, the picture just presented of decoherence, as due to classically stochastic environmental noise, may seem to disagree with the usual explanation in purely quantum language, according to which the different branches of a "Schrödinger's Cat state" excite orthogonal states of the environment 9].

Actually, the two explanations can be reconciled by realizing that our classical description effectively used a mixture of the Schrödinger picture for the Brownian system and the Heisenberg picture for the environment. It was thus able to refer only to the initial Wigner function of the environment, which was interpretable as a probability density. But if we were to use a uniformly Schrödinger picture, and follow the evolution of the entire Wigner function before integrating over the environmental sector, we would see that the negative values in the initial Wigner function of the system spread rapidly into the environmental sector. When the environmental integrals are performed, these contaminating oscillations into negative values provide cancellations that are the exact analogue of the vanishing inner products in the standard discussion. So the fact that the environment can affect the observed system only as a stochastic force does not allow us to conclude that the environment actually remains in a state described by a probabilistic ensemble.

Even if a Wigner function is positive definite, it still obeys a non-classical constraint associated with the Uncertainty Principle:

$$
\int d \Gamma f^{2} \leq 2 \pi \hbar
$$


where $\int d \Gamma$ denotes the integral over all of phase space. This constraint has important consequences. For example, a positive definite Wigner function has a well-defined Boltzman entropy, but this entropy will generally be larger than the von Neumann entropy. This can be interpreted as being due to the fact that nearby points in phase space are not distinct events in quantum mechanics, but are in a sense parts of the same object. So the von Neumann entropy represents a special kind of coarse-grained entropy.

(This idea provides a way to express the distinct quantum mechanical concepts of correlation and entanglement, in the Wigner representation. Compare the following two Wigner functions, each for a system of two oscillators:

$$
\begin{aligned}
& f_{1}=Z_{1} e^{-\frac{1}{m \Omega \hbar}\left(P_{1}^{2}+P_{2}^{2}\right)}\left[e^{-\frac{m \Omega}{\hbar}\left(Q_{1}-a\right)^{2}} e^{-\frac{m \Omega}{\hbar}\left(Q_{2}-a\right)^{2}}\right.\left.+e^{-\frac{m \Omega}{\hbar}\left(Q_{1}+a\right)^{2}} e^{-\frac{m \Omega}{\hbar}\left(Q_{1}+a\right)^{2}}\right] \\
& f_{2}=Z_{2} e^{-\frac{1}{m \Omega \hbar}\left(P_{1}^{2}+P_{2}^{2}\right)} e^{-\frac{m \bar{\Omega}}{\hbar}\left(Q_{1}^{2}+Q_{2}^{2}\right)} e^{\gamma Q_{1} Q_{2}} .
\end{aligned}
$$

Classically, one would say that both of these functions describe correlations between oscillators 1 and 2. But quantum mechanically, the first describes a correlation (for $\left.a^{2}>>\frac{\hbar}{m \Omega}\right)$, while the second describes an entanglement. We can conclude that entanglement is a correlation between parts of a single quantum mechanical object [10, and that the distinction between correlation and entanglement is not a separate piece of "quantum magic" from the uncertainty relation, but is actually a consequence of it.)

Despite the subtleties involved in interpreting Wigner functions, we emphasize that as long as one only uses classical intuition to understand how the classical equations of motion propagate them from initial to final times, there is nothing that can go wrong. This careful use of classical intuition can be of considerable value in appreciating the physics of linear quantum systems. One can expect to shed new light on the meaning of some important quantum mechanical effects, by identifying aspects of classical dynamics that are responsible for their generation. This is the good news, but it has a more pessimistic complement.

Because illustrations in linear models of basic quantum concepts are usually treated using techniques applicable to non-linear models as well, it is easy to receive an impression that these models are providing an insight into essentially quantum physics. It is important to realize, however, that the only specifically quantum aspects of any of these models reside in the initial conditions. To probe genuine quantum dynamics, we must accept the challenge of non-linearity, and must not confuse generality of formalism with generality of physics.

For their encouragement, and sharing of many insights, we would like to thank 
Bei-lok Hu (JA and SH), Emil Mottola, Bill Unruh, and Robert Zwanzig (SH). The support of the Natural Sciences and Engineering Research Council of Canada (JA) and the United States Department of Energy (SH) is gratefully acknowledged.

\section{References}

[1] See, e.g., M. Hillery, R. O'Connell, M. O. Scully, and E. P. Wigner, Phys. Rep. 106, 121 (1984); V. I. Tatarskii, Usp. Fiz. Nauk 139, 587 (1983) (Sov. Phys. Usp. 26, 311 (1983)).

[2] S. Habib, F. Cooper, and E. Mottola (in preparation).

[3] R. J. Rubin, J. Math. Phys. 1, 309 (1960); ibid 2, 373 (1961); G. W. Ford, M. Kac, and P. Mazur, J. Math. Phys. 6, 504 (1965); A. O. Caldeira and A. J. Leggett, Physica 121A, 587 (1983); H. Grabert, P. Schramm, and G.-L. Ingold, Phys. Rep. 168, 115 (1988).

[4] For discussions of the subtleties in defining "noise" in Langevin equations, see N. G. van Kampen, Stochastic Processes in Physics and Chemistry (North-Holland, Amsterdam, 1981); R. Zwanzig, in Systems Far From Equilibrium, edited by L. Garrido (Springer-Verlag, Berlin, 1980).

[5] J. R. Anglin and S. Habib, (in preparation).

[6] J. P. Paz, in The Physical Origin of Time Asymmetry, edited by J. J. Halliwell et al (Cambridge University Press, Cambridge, 1994).

[7] B. L. Hu, J. P. Paz, and Y. Zhang, Phys. Rev. D 45, 2843 (1992).

[8] P. M. V. B. Barone and A. O. Caldeira, Phys. Rev. A 43, 57 (1991); J. P. Paz, S. Habib, and W. H. Zurek, Phys. Rev. D 47, 488 (1993).

[9] See, e.g., W. H. Zurek, Phys. Today 44, No. 10, 36 (1991).

[10] A. Peres, Quantum Theory: Concepts and Methods (Kluwer Academic Publishers, Boston, 1993). 